\title{
Epidural Anesthesia for a Caesarean Delivery in a Parturient with an Intrathecal Baclofen Pump and Sickle Cell Disease: A Case Report
}

\author{
Bastian J", Bauer A, Kern C and Robertson-Baeriswyl M \\ Department of Anaesthesia, University Hospital of Lausanne, Switzerland

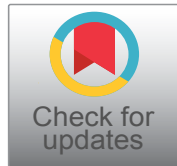

*Corresponding author: Bastian J, Department of Anaesthesia, University Hospital of Lausanne, Rue du Bugnon 21, 1011 Lausanne, Vaud, Switzerland, Tel: +410795562540, Fax: +41213142121

\begin{abstract}
A 32-year-old primigravida woman received an epidural anesthetic at 38 weeks of gestation for an elective caesarean delivery. She had for the past 10 years an intrathecal baclofen infusion pump to relieve spastic tetraparesis secondary to perinatal cerebral anoxia. She otherwise also suffered from sickle cell disease complicated by several vaso-occlusive crisis during pregnancy and a recent history of thromboembolic disease. A recent CT scan showed the intrathecal catheter entering the L3-L4 interspace and extending proximally to the thoracic region. For the caesarean delivery, an epidural catheter was sited using ultrasound in the L4-L5 interspace. Motor block and analgesia was obtained with a tested sensitive level never rising above T9. The patient was informed of the clinical situation and eventual risk of converting to general anesthesia if too uncomfortable during the caesarean section. Despite this she wished to forgo a general anesthesia if possible. It was decided to proceed with the caesarean delivery under epidural anesthesia. The caesarean section was uneventful, and no complications occurred in the post-natal period.

Neuraxial anesthesia may be favored over general anesthesia for patients with sickle cell disease, but epidural local anesthetic spread may be unpredictable following interventions involving the epidural space as illustrated by this case report.
\end{abstract}

\section{Keywords}

Caesarean section, Epidural anesthesia, Spastic tetraparesis, Intrathecal baclofen pump, Sickle cell anemia

\section{Abbreviations}

SCD: Sickle Cell Disease; ITB: Intrathecal Baclofen; GA: General Anesthesia

\section{Introduction}

The incidence of significant co-morbidities in the obstetric population is increasing [1]. Anaesthetists are therefore evermore confronted to a large range of conditions for which they need to be familiar with.

One of them could be the parturient with Sickle cell disease (SCD); as improved medical therapy and an increase in life expectancy over the last decades has led to a rise of such patients coming to child bearing age [2].

Another possibility could be the obstetric patient benefiting from intrathecal baclofen (ITB) therapy. ITB has been used since 1984 in patients with spasticity unresponsive to maximal doses of oral baclofen or other oral anti-spastic medications. Intrathecal baclofen pumps are now widely used to treat spasticity that is caused by a variety of aetiologies either spinal or cerebral in origin. The intrathecal route of administration is more efficacious and has a lower incidence of side effects than the oral route [3].

In recent years, two case reports have reported the successful use of epidural analgesia for labor in parturients with ITB with subsequent vaginal delivery $[4,5]$. None have though detailed the use of an epidural to provide a surgical anesthetic. In this case report, we describe the delivery of a surgical anesthetic provided with an epidural catheter for a caesarean section in a patient who combines those two significant and challenging comorbidities.

Citation: Bastian J, Bauer A, Kern C, Robertson-Baeriswyl M (2019) Epidural Anesthesia for a Caesarean Delivery in a Parturient with an Intrathecal Baclofen Pump and Sickle Cell Disease: A Case Report. Int J Anesthetic Anesthesiol 6:095. doi.org/10.23937/2377-4630/1410095

Accepted: August 21, 2019: Published: August 23, 2019

Copyright: (C) 2019 Bastian J, et al. This is an open-access article distributed under the terms of the Creative Commons Attribution License, which permits unrestricted use, distribution, and reproduction in any medium, provided the original author and source are credited. 


\section{Case Presentation}

A 32-year-old primigravida woman was assessed on request of the obstetrical team two months prior to an elective caesarean section planned at 38 weeks of gestation. She had been admitted to hospital for repeated vaso-occlusive crisis during the third trimester of her pregnancy due to her sickle cell disease, needing intensive pain management and regular blood transfusions. Following a recent pulmonary embolism, she was under therapeutic anticoagulation with heparin.

This patient was also benefiting from a continuous intrathecal baclofen pump at a dosage of $360 \mu \mathrm{g}$ per 24 hours implanted 10 years ago to relieve her spastic tetraparesis secondary to cerebral palsy. The pain specialist who had placed the ITB pump was consulted. A 20-gauge Medtronic intrathecal baclofen infusion catheter had been placed in the median position at the L3-L4 interspace with the tip in the mid-thoracic region. The rest of the catheter had been tunneled in the subcutaneous tissue lateral and inferior to the right costal margin and connected to the Medtronic pump situated in the abdominal subcutaneous tissue (Figure 1). A recent CT-scan was available to confirm these informations, and also showed the subcutaneous part of the tunnelled catheter passing over the median lumbar region from the L1 to L3 levels.

The female patient, $168 \mathrm{~cm}$ and $60 \mathrm{~kg}$ was wheelchair bound. A rigorous baseline neurological status was obtained, revealing spasticity and tetraparesis of all four limbs with a normal sensory exam. Examination of her back revealed a severe lumbar-dorsal kyphoscoliosis.
The rest of the pre-operative work-up was unremarkable. A transthoracic echocardiography showed normal biventricular function, and no pulmonary hypertension. Hemoglobin S level was at $18 \%$.

A multidisciplinary discussion took place among anesthesiologists, obstetricians, hematologists and the pain specialist who had placed the ITB pump. Her neurological condition raised concerns within the obstetrical team whether the parturient would be able to push effectively during labor to enable a straightforward vaginal delivery. It was thus concluded that an elective caesarean section at 38 weeks of gestation would be performed. Taking into account her co-morbidities, and after the patient's informed consent, it was chosen to do so under epidural anesthesia.

Intravenous heparin was stopped 4 hours prior to surgery and anti - Xa was checked and within normal range prior to bringing the patient to the operating theatre.

The patient was placed in the sitting position and ultrasonography of the L4-L5 interspace in the transverse and longitudinal views was performed. Skin to ligamentum flavum distance was estimated at $5 \mathrm{~cm}$ with the transverse view.

After sterile preparation and local anesthesia, an 18-gauge Tuohy needle was inserted at the L4-L5 interspace with a loss of resistance to saline effectively achieved at a depth of $5 \mathrm{~cm}$. An epidural catheter was then threaded $5 \mathrm{~cm}$ into the epidural space. After a negative test dose of $15 \mathrm{mcg}$ adrenaline with $30 \mathrm{mg}$ lidocaine, $15 \mathrm{ml}$ of $2 \%$ lidocaine in $5 \mathrm{ml}$ increments

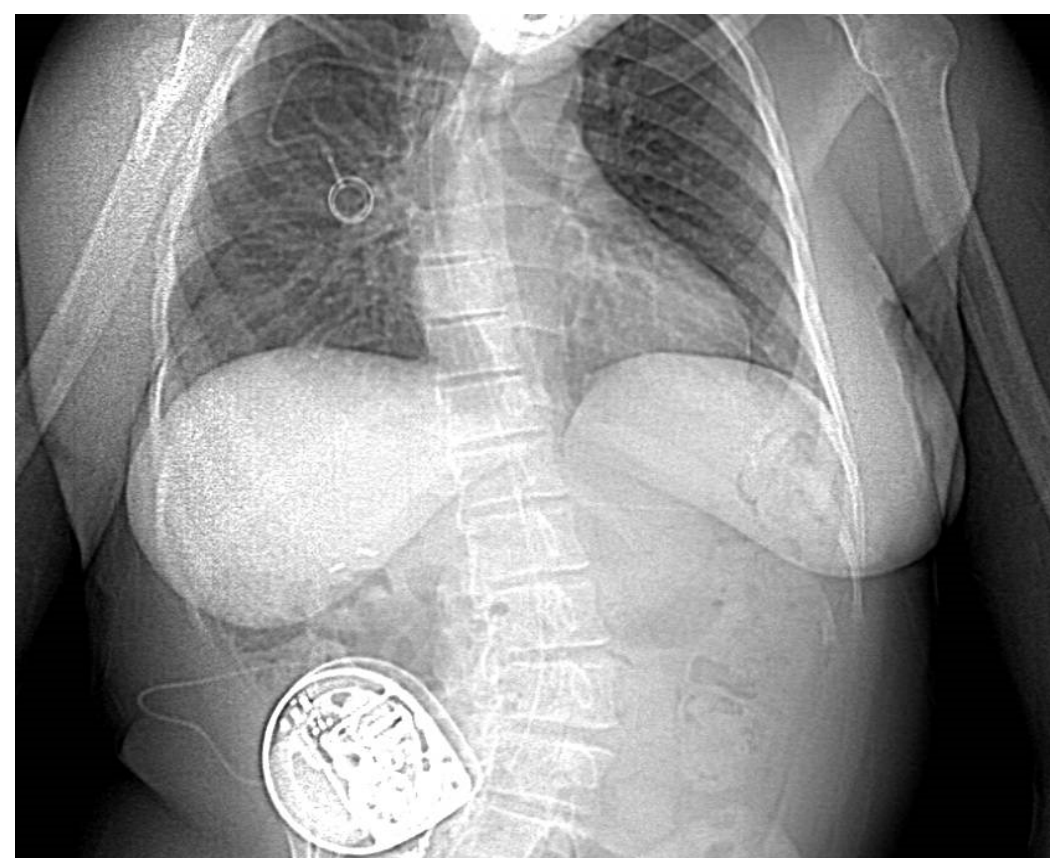

Figure 1: X-ray from a thoracoabdominal CT scan of the patient one month prior to the cesarean. Of note the severe kyphoscoliosis of the patient, the subcutaneous ITB pump in the right abdominal wall, and catheter seen to follow the lumbar column up to the thoracic region. 
were injected with 50 mcg fentanyl over 15 minutes. A continuous intravenous perfusion of neosynephrine was initiated according to our local protocol.

The patient developed rapidly good relaxation, loss of spasticity and a symmetrical and complete motor block of both legs. The sensitive block to cold was tested and found to be symmetrical at the T9 level. After another $15 \mathrm{~min}$, the sensitive level having not risen, increments of $5 \mathrm{ml}$ bupivacaine $0.5 \%$ were injected to a total of $10 \mathrm{ml}$ over 20 minutes. The sensitive level was again tested and still located at the T9 level. At that point, concerns were raised about systemic local anesthetic toxicity if more local anesthetic was injected into the epidural space. After discussion involving the obstetrician and anesthesiologist consultants, the patient was informed of the clinical situation and the eventual risk of having to convert to a general anesthesia if uncomfortable during the cesarean section under epidural anesthesia. The patient absolutely wishing to forgo a general anesthesia (GA) if possible, it was decided to proceed with the cesarean delivery under epidural anesthesia. The cesarean section was uneventful with uterotomy and birth occurring 9 min after incision. An intravenous dose of $5 \mathrm{UI}$ of oxytocin over $5 \mathrm{~min}$ followed by a continuous perfusion of $0.8 \mathrm{UI} / \mathrm{h}$ was given following umbilical cord clamping with good clinical effect on the uterus. The newborn adapted well to extra uterine life with an Apgar score of 5/7/10 and the mother was comfortable throughout the entire operation. Total estimated blood loss was $600 \mathrm{ml}$. Post-operative analgesia was managed with paracetamol and morphine.

The patient was discharged home seven days postpartum without complaints.

At one year, the patient was followed up, both the mother and child are doing well.

\section{Discussion}

During pregnancy and the postpartum, women with SCD are known to be at increased risk for complications such as vaso-occlusive crisis, acute chest syndrome, infection and thrombo-embolic events [2]. A retrospective study evaluating the impact of anesthetic technique on the occurrence of postnatal sickling complications determined GA to be a probable risk factor for postnatal sickling, and vaso-occlusive crisis [6]. Regional anesthesia may have on the other hand, potential beneficial effects. Firstly, sympathetic blockade, vasodilatation and venous blood pooling enhances blood flow in the anaesthetized area. Secondly, it provides optimal control of pain as proved by reports of vaso-occlusive crisis during labor being successfully managed using neuraxial analgesia [7]. Thirdly, neuraxial anesthesia decreases the risk of maternal pulmonary aspiration that might occur during $G A$, inducing hypoxia and precipitation of acute chest syndrome in parturients with SCD.

Whilst GA for cesarean delivery is associated with a significantly increased risk of venous thromboembolism [8], neuraxial anesthesia through attenuation of the sympathetic response to surgery, improved venous blood flow and anticoagulant properties of local anesthetics, improves components of the Virchow's triad which are pathogenic contributors to venous thrombosis [9].

For those aforementioned reasons, we favored neuraxial anesthesia over GA in our patient with a history of homozygous SCD and recent pulmonary embolism.

Although the patient was under therapeutic intravenous heparin for her recent pulmonary embolism, neuraxial anesthesia was deemed feasible as the cesarean would be elective. Appropriate measures could be taken to minimize the risk of epidural hematoma. According to local protocol, heparin could be stopped 4 hours prior epidural insertion, and any residual activity of heparin by dosing anti Xa activity could be checked, prior to engaging with the procedure. The occurrence of epidural hematomas being also linked to the numbers of punctures and attempts to site an epidural catheter, we favored the use of ultrasonography so as to increase success, ease of performance and reduction in traumatic procedures.

Due to lack of clinical evidence or literature, many anesthesiologists would probably consider the presence of an intrathecal pump as a relative contraindication when considering neuraxial anesthesia. The ITB pump raised several concerns for the peri-operative period. First, the physical presence of the pump and catheter raised concerns about damage to the ITB catheter when the epidural needle would be inserted.

Our review of the literature found several case reports of pregnancy in women with ITB therapy [10]. Two of them describe epidural placement in patients with ITB pumps for labor analgesia with subsequent successful vaginal delivery. The first was receiving ITB therapy for spasticity associated with cerebral palsy, the epidural was sited in the L4-L5 interspace using anatomical landmarks, one level below the intrathecal catheter insertion site [4]. The epidural provided good analgesia. Labor and delivery were uneventful. In the second case report, a parturient with an ITB pump for left spastic hemiplegia received epidural analgesia using ultrasonography to guide insertion. The epidural was placed at the first attempt and labor continued normally [5]. No case reports up until now describes the use of epidural anesthesia for caesarean section in a parturient with an ITB pump.

We chose to place the epidural catheter in the L4L5 interspace one level below the intrathecal infusion catheter because concerns were raised that damage might occur to the subcutaneously tunneled catheter passing over the median aspect of the lumbar region from L1 to L3 should we choose to site the epidural 
above the L3-L4 insertion level. Insertion at a level above L1 may have prevented proper anesthesia of the sacral nerve roots, and the aforementioned case report [4] also successfully sited the epidural catheter one level below the intrathecal catheter without any complications. The landmark technique for locating vertebral interspace is known to be imprecise with up to $71 \%$ of failure rate when identifying a given lumbar interspace [11]. Use of ultrasonography is linked with an increased success, ease of performance and reduction in traumatic procedures [12]. In our patient, we used ultrasonography to locate the L4-L5 interspace, skin to ligamentum flavum distance, best angle for epidural needle insertion.

Although the surgical anesthesia provided by the epidural catheter allowed an uneventful caesarean section, the epidural block never rose above the T9 level despite appropriate local anesthetic dosing. There is no mention in the literature of the effect on the epidural space of the long-term presence of a foreign body such as an ITB catheter. It is reasonable however to hypothesize that the continued presence of a foreign body in the epidural space at the level of L3/L4 may have resulted in scarring and thus be responsible for the poor cephalad spread.

Our second hypothesis concerns the patient's severe lumbar-dorsal kyphoscoliosis. Severe scoliosis has been shown to alter epidural anesthetic spread [13]. It is possible to imagine that her spinal deformity with severe lumbar and dorsal curves and the change in position after insertion of the epidural catheter might have prevented the spread of local anesthetic.

Another hypothesis would be anatomical, as reports in the literature mention fibrous bands of connective tissue named Hofmann's ligaments mainly in the lumbar epidural space, that link the dural sac to the posterior longitudinal ligament, the vertebral canal and the ligamentum flavum [14]. This seems unlikely though, as evaluation of postmortem spread of epidural injectate of ink in humans using cryomicrotome imaging found no substantial barriers to the distribution of ink inside the epidural space, apart from the fascia of the posterior longitudinal ligament [15].

Our use of an intravenous perfusion of neosynephrine to mitigate the hypotension induced by epidural anesthesia and to ensure adequate placental perfusion can be debated. Vasopressors such as epinephrine, through their vasoconstrictive effect, stasis and activation of red cell adhesion to the endothelium, have been speculated to increase the risk of sickling and therefore of vaso-occlusion [16]. On the other hand, ephedrine was not identified as a risk factor for postnatal sickling complications in a retrospective study [6]. No data was found for neosynephrine. Our patient did not develop symptoms suggesting vaso-occlusive crisis in the per or post-operative period.
Baclofen is a chemical derivative of the inhibitory neurotransmitter Gamma-Amino butyric Acid (GABA). Baclofen inhibits sensory input to the motor neurons through a selective binding and activation of GABA-B receptors in the spinal cord both at presynaptic sites by decreasing calcium conduction resulting in decreased excitatory amino acid glutamate and aspartate release, and postsynaptic sites by increasing potassium conductance leading to neuronal hyperpolarization [17]. Intrathecal baclofen is best continued in the peri partum period as abrupt cessation of baclofen can trigger baclofen withdrawal which may be life threatening $[3,18]$. Symptoms may start within the first hours and up to 48-72 hours after abrupt cessation. They range from initially mild symptoms such as lightheadedness, dizziness, anxiety, worsening or return of spasticity, but can progress to hyperthermia, seizures, muscle rigidity, rhabdomyolysis, acute kidney injury, disseminated intravascular coagulation, multisystem organ failure, cardiac arrest, coma and death $[3,18]$. Our patient was reassessed regularly in the following days after the procedure for any new symptoms or signs which would have enabled us to promptly identify a malfunction of the ITB catheter or pump. Fortunately, our patient remained asymptomatic.

\section{Conclusion}

This case should serve as an important reminder that the spread of epidural analgesia or anesthesia is unpredictable in patients who have had interventions involving the epidural space and/or severe kyphoscoliosis. In retrospect, neuraxial anesthesia was a good option for this patient considering her sickle cell disease and her recent history of pulmonary embolism. We could probably have considered more strongly the option of a spinal anesthetic, as it would have guaranteed a better cephalad spread of the local anesthetic and thus an adequate sensitive and motor block.

\section{References}

1. Hess PE (2017) What's new in obstetric anesthesia: The 2016 Gerard W. Ostheimer lecture. Anesth Analg 124: 863871.

2. Villers MS, Jamison MG, De Castro LM, James AH (2008) Morbidity associated with sickle cell disease in pregnancy. Am J Obstet Gynecol 199: 125.

3. Woolf SM, Baum CR (2017) Baclofen Pumps: Uses and Complications. Pediatr Emerg Care 33: 271-275.

4. Ali Sakr Esa W, Toma I, Tetzlaff JE, Barsoum S (2009) Epidural analgesia in labor for a woman with an intrathecal baclofen pump. Int J Obstet Anesth 18: 64-66.

5. Badve M, Shah T, Jones-Ivy S, Vallejo MC (2011) Ultrasound guided epidural analgesia for labor in a patient with an intrathecal baclofen pump. Int J Obstet Anesth 20: 370-371.

6. Camous J, N'da A, Etienne-Julan M, Stéphan F (2008) Anesthetic management of pregnant women with sickle cell disease-effect on postnatal sickling complications. Can J Anaesth 55: 276-283. 
7. Danzer BI, Birnbach DJ, Thys DM (1996) Anesthesia for the parturient with sickle cell disease. J Clin Anesth 8: 598602.

8. Guglielminotti J, Landau R, Li G (2019) Adverse events and factors associated with potentially avoidable use of general anesthesia in cesarean deliveries. Anesthesiology 130: 912-922.

9. Turan A, Bajracharya GR, Leung S, Yazici Kara M, Mao $G$, et al. (2018) Association of neuraxial anesthesia with postoperative venous thromboembolism after noncardiac surgery: A propensity-matched analysis of ACS-NSQIP database. Anesthesia \& Analgesia 128: 494-501.

10. Takeshi Hara, Madoka Nakajima, Hidenori Sugano, Kostadin Karagiozov, Emiko Hirose, et al. (2018) Pregnancy and breastfeeding during intrathecal baclofen therapy a case study and review. NMC Report 5: 65-68.

11. Broadbent CR, Maxwell WB, Ferrie R, Wilson DJ, Gawne Cain M, et al. (2000) Ability of anaesthetists to identify a marked lumbar interspace. Anaesthesia 55: 1122-1126.

12. Perlas A, Chaparro LE, Chin Ki J (2016) Lumbar neuraxial ultrasound for spinal and epidural anesthesia: A systematic review and meta-analysis. Reg Anesth Pain Med 41: 251-260.

13. CB Collier (2014) Neuraxial anaesthesia in patients with scoliosis. BJA 112: 1125-1126.

14. Gabrielle G Tardieu, Christian Fisahn, Marios Loukas, Marc Moisi, Jens Chapman, et al. (2016) The epidural ligaments (of Hofmann): A comprehensive review of the literature. Cureus 8: e779.

15. Quinn Hogan (2002) Distribution of Solution in the epidural space: Examination by cryomicrotome section. Reg Anesth Pain Med 27: 150-156.

16. Zennadi R, Hines PC, De Castro LM, Cartron JP, Parise LV, et al. (2004) Epinephrine acts through erythroid signaling pathways to activate sickle cell adhesion to endothelium via LW-alphavbeta3 interactions. Blood 104: 3774-3781.

17. Bottros MM, Christo PJ (2014) Current perspectives on intrathecal drug delivery. J Pain Res 7: 615-626.

18. Imran Mohammed, Asif Hussain (2004) Intrathecal baclofen withdrawal syndrome a life-threatening complication of baclofen pump: A case report. BMC Clin Pharmacol 4: 6. 